\title{
Redesigning the NEDD8 pathway with a bacterial genetic screen for ubiquitin-like molecule transfer
}

\author{
Gurkan Guntas and Brian Kuhlman \\ Department of Biochemistry and Biophysics, University of North Carolina, Chapel Hill, North \\ Carolina 27599-7260, USA
}

\begin{abstract}
Pathways of ubiquitin-like (UBL) molecule transfer regulate a myriad of cellular cascades. Here we report a high-throughput assay that correlates catalytic human-NEDD8 transfer to bacterial survival. The assay was utilized to screen mutant NEDD8 and NEDD8-activating enzyme (NAE) libraries to engineer a more stable NEDD8 and redesign the NEDD8-NAE interface. This approach will be useful in understanding the specificities underlying UBL pathways.
\end{abstract}

\section{Introduction}

Ubiquitin and ubiquitin-like-molecule (UBLs, i.e. NEDD8, SUMO) transfer is a key posttranslational modification mechanism that controls protein abundance and cell regulatory elements. The transfers of UBLs to target substrates are mediated by their respective enzymatic pathways that involve cognate UBL-activating (E1s) and UBL-conjugating enzymes (E2s). For example, APPBP1-UBA3 heterodimer (NAE) specifically catalyzes NEDD8-activation and transfers NEDD8 to the active site cysteine residue of the NEDD8conjugating enzyme, Ubc12 $1 ; 2$. NEDD8-charged Ubc12 then catalyzes the transfer of NEDD8 onto its specific substrates. For ubiquitin, the pathway is more complex as it involves multiple E2s that interact with more than 600 different E3-ligase enzymes to transfer ubiquitin to target substrates ${ }^{3}$. The specificity determinants of the interactions among UBLs and the enzymes are not completely determined.

Practical high-throughput screens that report UBL-transfer would be very useful for probing sequence-structure-function relationships in these pathways. To that end, we coexpressed modified versions of NEDD8, Ubc12, and NAE to reconstitute the neddylation pathway in E.coli in order to establish a genetic selection that is able to probe the catalytic activation and transfer of NEDD8 onto Ubc12. Ubc12 was fused toa variant of $\beta$-lactamase(BLA) that is missing its signaling sequence for export to the periplasm and NEDD8 to the ssTorA (signal sequence for Trimethylamine-N-oxide reductase 1) bacterial signal peptide that is responsible for exporting well-folded proteins to the perisplamic compartment of gramnegative bacteria ${ }^{4 ; 5 ; 6 ; 7}$. Since, penicillin based antibiotics attack the cell wall of bacteria, $\beta$-lactamase needs to be exported to the periplasm toconfer resistance to such antibiotics. In our scheme (Fig. 1a), the catalytic formation of the NEDD8-Ubc12 thioester bond provides a link between the ssTorA signal peptide and $\beta$-lactamase and confers antibiotic resistance to

(C) 2012 Elsevier Ltd. All rights reserved.

Correspondence to: Brian Kuhlman.

Publisher's Disclaimer: This is a PDF file of an unedited manuscript that has been accepted for publication. As a service to our customers we are providing this early version of the manuscript. The manuscript will undergo copyediting, typesetting, and review of the resulting proof before it is published in its final citable form. Please note that during the production process errors may be discovered which could affect the content, and all legal disclaimers that apply to the journal pertain. 
the cell. Further transfer of the NEDD8 to a lysine on Ubc12 via autoneddylation would also provide antibiotic resistance.

\section{A bacterial screen for NEDD8 transfer}

We tested the validity of neddylation dependent localization by constructing a plasmid that encodes all the genes of interest (pNEDD8, Fig. 1b). The NEDD8 gene is preceded by the ssTorA signal sequence and a FLAG peptide. In this construct, the N-terminal methionine of NEDD8, is replaced by a threonine (called NEDD8 ${ }^{\mathrm{M} 1 \mathrm{~T}}$ ).BLA was fused to the $\mathrm{C}$-terminus of Ubc12.E.coli harboring pNEDD8 is able to form colonies on solid media supplemented with ampicillin (Fig. 1c). As negative controls, we tested two plasmids in which either NEDD ${ }^{\mathrm{M} 1 \mathrm{~T}}$ gene was replaced with ubiquitin or a mutation was introduced rendering the NAE inactive. In both cases, transformed cells were killed by low levels of ampicillin. Ubiquitin, despite having $56 \%$ sequence identity with NEDD8, is not recognized by the neddylation cascade. Much of the specificity stems from residue 72. Ubiquitin and NEDD8 possess arginine and alanine at this position respectively. It has been shown that Ubiquitin_R72A mutant is recognized as a substrate by the neddylation enzymes $8 ; 9 ; 10$. Consistent with this view, introducing this mutation into ubiquitin rescued ampicillin resistance (Fig. 1c).

Although the ssTorA-NEDD8-Ubc12-BLA conjugate is export- and ampicillin resistancecompetent, several other species are expected to be exported to the periplasm. First, translocation of ssTorA-NEDD8 and/or ssTorA-NEDD8-NAE is expected to compete against the transthiolation reaction by decreasing the available NEDD8 concentration. Second, ssTorA-NEDD8-NAE needs to bind and form a transient complex with Ubc12 before catalyzing the transthiolation reaction. However, this complex is unlikely to result in false positive growth because it is large, has a short lifetime, and NAE expression in bacteria is low relative to Ubc12 and BLA. In addition, the N-terminal tail of Ubc12 was not included in our construct, which results in about 26-fold increase in $\mathrm{Km}$ for the transfer of NEDD8 from NAE to Ubc12 ${ }^{11}$. Finally, the active site mutant of NAE abolished ampicillin resistance (Fig. 1c) suggesting that transient interactions are not sufficient to create an ampicillin resistance phenotype.

\section{Stabilizing NEDD8}

As an initial test whether a high-throughput screen with our system is feasible we screened for NEDD8 ${ }^{\mathrm{M} 1 \mathrm{~T}}$ mutations that increase ampicillin resistance. Previously, we have observed that wild type NEDD8 does not express robustly in E. coli $(\sim 5 \mathrm{mg} / \mathrm{liter})$, and NEDD8 ${ }^{\mathrm{M} 1 \mathrm{~T}}$ expresses at even lower levels $(<1 \mathrm{mg} /$ liter $)$, suggesting that mutations that increase NEDD8 ${ }^{\mathrm{M} 1 \mathrm{~T}}$ expression may lead to higher levels of Ubc12 conjugated with NEDD8 ${ }^{\mathrm{M} 1 \mathrm{~T}}$. The library of NEDD8 mutants were created by error-prone PCR based random mutagenesis with NEDD8 ${ }^{\mathrm{M} 1 \mathrm{~T}}$ as the starting sequence. After three rounds of selection at nonpermissive ampicillin concentrations ( 64 and $128 \mu \mathrm{g} / \mathrm{mL}$ ), six clones with improved ampicillin resistances were identified (Fig. 2a). All six clones contained the same double mutation (L8P and Q49L) suggesting that at least these two mutations are required for much of the observed improvement. Clone M6 that only contained the double mutation was characterized further.

The NEDD8 mutations found in clone M6 may be raising ampicillin resistance by a variety of mechanisms including an increase in protein expression or faster kinetic parameters for E1 activation or E1 / E2 transthiolation. In vitro experiments with equal quantities of purified proteins showed similar rates of Ubc12 conjugation with NEDD8 and M6, suggesting that the kinetic parameters were not significantly perturbed for M6 (Fig. 2b). More dramatic were the changes in protein expression, the yield from a 1 liter culture was 
$\sim 5$-fold greater for M6 compared to NEDD8 ${ }^{\mathrm{M} 1 \mathrm{~T}}$. In some cases, protein expression is linked to the thermodynamic stability of a protein. Chemical denaturation experiments showed that NEDD8 ${ }^{\mathrm{M} 1 \mathrm{~T}}$ is only marginally stable at $20^{\circ} \mathrm{C}$, with a free energy of unfolding of $<1 \mathrm{kcal} /$ mol, while M6 has a stability of $2.4 \mathrm{kcal} / \mathrm{mol}$. Additionally, NEDD8 ${ }^{\mathrm{M} 1 \mathrm{~T}}$ unfolds at lower temperatures than M6 in thermal denaturation experiments(Fig. 2c). Both M6 and NEDD8 ${ }^{\mathrm{M} 1 \mathrm{~T}}$ unfold irreversibly when heat denatured, indicating that changes in thermal stability may impact expression levels as proteins that stochastically unfold are removed from the pool of active molecules. The two mutations in M6 also increase the thermostability of wild type NEDD8 (1.8 and $3.2 \mathrm{kcal} /$ mol respectively, Fig S2). Consistent with the difference in in vitro stabilities, replacing the N-terminal HMDYKDDDDK-GT sequence of NEDD8 ${ }^{\mathrm{M} 1 \mathrm{~T}}$ with HM-GT-M (restores the N-terminal methionine of NEDD8)increases the levels of ampicillin resistance by $\sim 4$-fold.One attractive feature of our screen is that because it depends on multiple stages of the neddylation pathway, it identified mutations that increase NEDD8 stability without compromisingNEDD8 functionality.

\section{Creating an altered specificity NEDD8 NAE interaction}

NEDD8 and ubiquitin are 56\% identical and their respective activating enzymes are $43 \%$ identical. Despite these close similarities, NEDD8 and ubiquitin are considerably more reactive with their cognate E1 enzymes than the off-pathway E1s. Much of the specificity has been mapped to one residue on either protein. Several mutations at position 72 of NEDD8 (A72Q, A72K, A72R) and of ubiquitin (R72A, R72L) have been examined for specificity $8 ; 9 ; 10 ; 12$.NEDD8_A72Q and ubiquitin_R72L have been shown to be substrates for NAE suggesting that $\mathrm{L}$ and $\mathrm{Q}$ are tolerated at this position. To search for additional tolerated mutations, we performed site-saturation mutagenesis at position 72 of NEDD8 by constructing a small library (16 members excluding proline, alanine, arginine, and glycine). After 2 rounds of selection, we sequenced 13 members of the final survivor pool. Leucine, glutamine, and methionine were found four times each and threonine was found once. These results were validated by spot test assays and by in vitro neddylation assays (Fig. 3a). In line with the selection results, among the new mutants tested only NEDD8_A72M was a competent substrate for NAE in transfer assays.

Encouraged by the screen's ability to select for functional NAE-NEDD8 pairs, we wondered whether it would be possible to engineer pathways that are orthogonal to the existing UBL pathways. Based on the crystal structure of NEDD8 in complex with NAE ${ }^{1}$, we selected 5 NAE residues that are proximal to NEDD8_A72. A library of NAE mutants where all 20 amino acids were allowed at those five positions was screened against NEDD8_A72(F/Y/W/ C/S). These NEDD8 variants were targeted because they did not show activity with wild type NAE and large aromatic residues can create strong steric clashes useful for creating specificity. After three rounds of selection, three NAE/NEDD8 pairs were sequenced and their growth was validated with independent assays (Supplementary Table 1). The sequence similarities among the NAE clones suggested that the final pool had limited sequence diversity while all NEDD8 clones possessed tyrosine at position 72 . Spot tests verified that the NAE2/NEDD8_A72Y pair helped cells confer as much ampicillin resistance as the wt/ wt pair and significantly more resistance than the wtNAE/NEDD8_A72Y pair (Supplementary Figure 1). In vitro Ubc12 transthiolation experiments also showed that the NAE2/NEDD8_A72Y pair was more active than the wtNAE/NEDD8_A72Y pair, and although it was not explicitly selected for, NAE2 showed reduced activity with wild-type NEDD8.

The NEDD8-transthiolation mediated localization allows screening libraries for functional proteins of the neddylation pathway. This strategy is well suited to implement selection 
platforms for the pathways of other ubiquitin-like molecules. The transfer pathways for $\mathrm{SUMO}^{13} ; 14 ; 15$, ubiquitin ${ }^{16}$, and Atg $8^{17}$ have previously been reconstituted in E.coli, suggesting that they are amenable to our strategy. Furthermore, in particular for ubiquitination, the screen can be expanded to include E3 ligases and their substrates. Such an approach could help identify the specificity determining factors among the E3 ligase substrate pairs. Also, the positive selection described here can potentially be combined with a previously described negative screening for $\beta$-lactamase activity ${ }^{18}$. Such dual selections might allow complete elucidation of sequence-function relationships for ubiquitin and ubiquitin-like pathways.

\section{Supplementary Material}

Refer to Web version on PubMed Central for supplementary material.

\section{Acknowledgments}

We would like to thank Matt DeLisa for providing us the pSalect vector and Brenda Schulman for providing us pGEX-4T-1-Ubc12 and pGEX-4T-1-APPBP1-UBA3 vectors.

\section{References}

1. Huang DT, Hunt HW, Zhuang M, Ohi MD, Holton JM, Schulman BA. Basis for a ubiquitin-like protein thioester switch toggling E1-E2 affinity. Nature. 2007; 445:394-8. [PubMed: 17220875]

2. Walden H, Podgorski MS, Schulman BA. Insights into the ubiquitin transfer cascade from the structure of the activating enzyme for NEDD8. Nature. 2003; 422:330-4. [PubMed: 12646924]

3. Deshaies RJ, Joazeiro CA. RING domain E3 ubiquitin ligases. Annu Rev Biochem. 2009; 78:399434. [PubMed: 19489725]

4. DeLisa MP, Samuelson P, Palmer T, Georgiou G. Genetic analysis of the twin arginine translocator secretion pathway in bacteria. J Biol Chem. 2002; 277:29825-31. [PubMed: 12021272]

5. DeLisa MP, Tullman D, Georgiou G. Folding quality control in the export of proteins by the bacterial twin-arginine translocation pathway. Proc Natl Acad Sci U S A. 2003; 100:6115-20. [PubMed: 12721369]

6. Fisher AC, Kim W, DeLisa MP. Genetic selection for protein solubility enabled by the folding quality control feature of the twin-arginine translocation pathway. Protein Sci. 2006; 15:449-58. [PubMed: 16452624]

7. Weiner JH, Bilous PT, Shaw GM, Lubitz SP, Frost L, Thomas GH, Cole JA, Turner RJ. A novel and ubiquitous system for membrane targeting and secretion of cofactor-containing proteins. Cell. 1998; 93:93-101. [PubMed: 9546395]

8. Souphron J, Waddell MB, Paydar A, Tokgoz-Gromley Z, Roussel MF, Schulman BA. Structural dissection of a gating mechanism preventing misactivation of ubiquitin by NEDD8's E1. Biochemistry. 2008; 47:8961-9. [PubMed: 18652489]

9. Walden H, Podgorski MS, Huang DT, Miller DW, Howard RJ, Minor DL Jr, Holton JM, Schulman BA. The structure of the APPBP1-UBA3-NEDD8-ATP complex reveals the basis for selective ubiquitin-like protein activation by an E1. Mol Cell. 2003; 12:1427-37. [PubMed: 14690597]

10. Whitby FG, Xia G, Pickart CM, Hill CP. Crystal structure of the human ubiquitin-like protein NEDD8 and interactions with ubiquitin pathway enzymes. J Biol Chem. 1998; 273:34983-91. [PubMed: 9857030]

11. Huang DT, Miller DW, Mathew R, Cassell R, Holton JM, Roussel MF, Schulman BA. A unique E1-E2 interaction required for optimal conjugation of the ubiquitin-like protein NEDD8. Nat Struct Mol Biol. 2004; 11:927-35. [PubMed: 15361859]

12. Bohnsack RN, Haas AL. Conservation in the mechanism of Nedd8 activation by the human AppBp1-Uba3 heterodimer. J Biol Chem. 2003; 278:26823-30. [PubMed: 12740388]

13. Mencia M, de Lorenzo V. Functional transplantation of the sumoylation machinery into Escherichia coli. Protein Expr Purif. 2004; 37:409-18. [PubMed: 15358364] 
14. Uchimura Y, Nakamura M, Sugasawa K, Nakao M, Saitoh H. Overproduction of eukaryotic SUMO-1- and SUMO-2-conjugated proteins in Escherichia coli. Anal Biochem. 2004; 331:204-6. [PubMed: 15246018]

15. Uchimura Y, Nakao M, Saitoh H. Generation of SUMO-1 modified proteins in E. coli: towards understanding the biochemistry/structural biology of the SUMO-1 pathway. FEBS Lett. 2004; 564:85-90. [PubMed: 15094046]

16. Rosenbaum JC, Fredrickson EK, Oeser ML, Garrett-Engele CM, Locke MN, Richardson LA, Nelson ZW, Hetrick ED, Milac TI, Gottschling DE, Gardner RG. Disorder targets misorder in nuclear quality control degradation: a disordered ubiquitin ligase directly recognizes its misfolded substrates. Mol Cell. 41:93-106. [PubMed: 21211726]

17. Ichimura Y, Imamura Y, Emoto K, Umeda M, Noda T, Ohsumi Y. In vivo and in vitro reconstitution of Atg8 conjugation essential for autophagy. J Biol Chem. 2004; 279:40584-92. [PubMed: 15277523]

18. Sohka T, Heins RA, Phelan RM, Greisler JM, Townsend CA, Ostermeier M. An externally tunable bacterial band-pass filter. Proc Natl Acad Sci U S A. 2009; 106:10135-40. [PubMed: 19502423]

19. Lutz S, Fast W, Benkovic SJ. A universal, vector-based system for nucleic acid reading-frame selection. Protein Eng. 2002; 15:1025-30. [PubMed: 12601143]

20. Cadwell RC, Joyce GF. Randomization of genes by PCR mutagenesis. PCR Methods Appl. 1992; 2:28-33. [PubMed: 1490172]

21. Huang DT, Schulman BA. Expression, purification, and characterization of the E1 for human NEDD8, the heterodimeric APPBP1-UBA3 complex. Methods Enzymol. 2005; 398:9-20.

[PubMed: 16275315] 


\section{Highlights}

- Reconstituted the neddylation pathway in bacteria

- Ampicillin resistance was coupled to catalytic transfer of NEDD8

- Used selection scheme to identify mutations that stabilize NEDD8.

- Engineered an altered specificity interface between NEDD8 and its E1 enzyme 

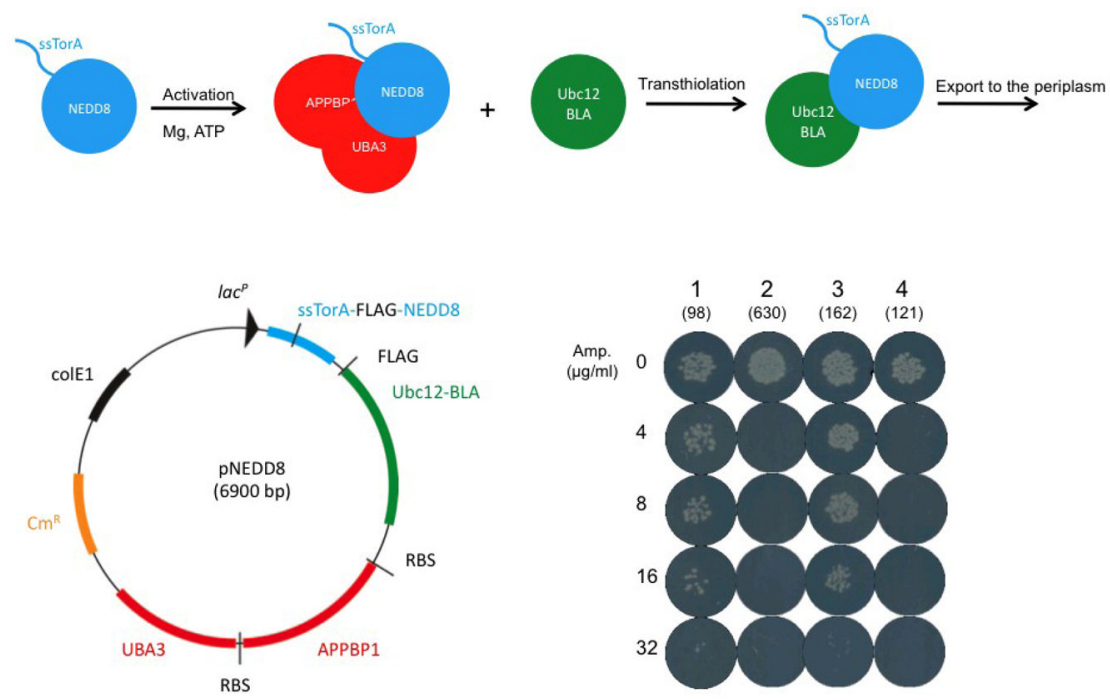

Figure 1.

The strategy for establishing a link between E.coli survival and Ubc12-NEDD8 transthiolation.(A) NEDD8 with an N-terminal signal sequence, ssTorA, is activated by NAE and transferred onto the active site cysteine of Ubc12 that is expressed as a fusion to $\beta$ lactamase. The whole complex is exported from the cytoplasm to the periplasm where $\beta$ lactamase is active and confers cells ampicillin resistance. (B) The map of the expression plasmid. RBS: Ribosome binding site, $\mathrm{Cm}^{\mathrm{R}}$ : Chloramphenicol resistance gene. Genes encoding for DYKDDDDK-GT-NEDD8_[Leu2-Gly76] and DYKDDDDK-MetUBC12_[Ala29-183Lys]-BLA_[His24-Trp286] fusions separated by stop codons were in tandem cloned into pSalect vector ${ }^{19}$ via NdeI/EcorI sites. The first methionine of NEDD8 and the N-terminal tail of UBC12 were replaced with the FLAG (DYKDDDDK) - GT and FLAG peptides respectively. (C) Levels of ampicillin resistance of cells carrying different versions of the plasmid. 1: plasmid shown in (B), 2: NEDD8 gene is replaced with ubiquitin, 3: Ubiquitin_R72A that can mimic NEDD8. 4: NAE_C237A inactive mutant. Numbers in parentheses indicate the number of estimated cells in the spot. Overnight cultures of isolated MC4100 colonies transformed with plasmids of interest were diluted to OD600 of 0.001 and $5 \mu \mathrm{l}$ of the diluted stocks were spotted on LB agar plates supplemented with $25 \mu \mathrm{g} / \mathrm{ml}$ Chloramphenicol and varying concentrations of ampicillin. Growth was documented after two days of incubation at room temperature. In order to estimate the number of cells in a given spot, $5 \mu$ of diluted cells were spread on LB agar plates supplemented with only 25 $\mu \mathrm{g} / \mathrm{ml}$ Chloramphenicol and a colony count was performed after two days of incubation at room temperature. 
A)

\begin{tabular}{|c|c|}
\hline Mutant & Mutations \\
\hline M1 & L8P, Q49L, S46G \\
\hline M2 & L8P, Q49L, R42K \\
\hline M3, M4, M6 & L8P, Q49L \\
\hline M5 & L8P, Q49L, E12D \\
\hline
\end{tabular}

B)

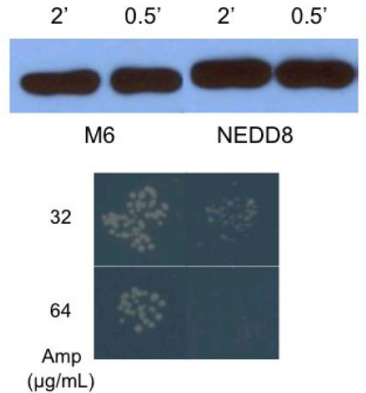

C)
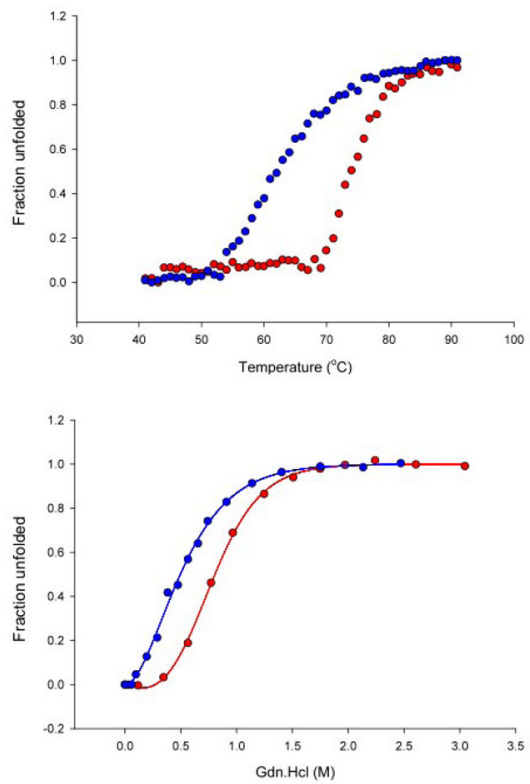

Figure 2.

The screen identifies a mutant of NEDD8 with enhanced stability. (A) Sequences of selected mutants. (B) Western blot of Ubc12 conjugated in vitro with either M6 or w.t. NEDD8. Spot test comparison of cells expressing M6 $6^{\mathrm{M} 1 \mathrm{~T}}$ and $\mathrm{NEDD}^{\mathrm{M} 1 \mathrm{~T}}$ demonstrating the increase in ampicillin resistance. $(\mathbf{C})$ Thermal and chemical denaturation of $\mathrm{M}^{\mathrm{M} 1 \mathrm{~T}}$ (red) and $\mathrm{NEDD}^{\mathrm{M} 1 \mathrm{~T}}$ (blue).

To create a library of NEDD8 variants NEDD8 was PCR-amplified under mutagenic conditions as described ${ }^{20}$ and cloned into pNEDD8 to yield about $6.8 \times 10^{6}$ independent transformants. For the first round of selection, $10^{6}$ colony forming units were plated on two $245 \mathrm{~mm} \times 245 \mathrm{~mm}$ LB/agar plates supplemented with $25 \mu \mathrm{g} / \mathrm{ml}$ Chloramphenicol and $64 \mu \mathrm{g} /$ $\mathrm{ml}$ of ampicillin. Genetic material from $\sim 10^{4}$ survivors was PCR-amplified and cloned back into pNEDD8 for further selection. After 2 more rounds of selection at 64 and $128 \mu \mathrm{g} / \mathrm{ml}$ of ampicillin, 6 clones were sequenced. All proteins were expressed in BL21(DE3)pLysS cells as C-terminal fusions to Glutathione S-Transferase (GST) and purified initially by GSTaffinity chromatography. NEDD8 variants with [GS-DYKDDDDK-GGSGG-Met1_Gly76] or without [GS-Leu2_Gly76] the FLAG epitope were further purified by S75 size-exclusion chromatography after site-specific cleavage by thrombin to remove the GST tag. After thrombin digestion, full-length Ubc12 was purified by cation exchange chromatography. Transthiolation reactions were carried out at room temperature in the presence of $4 \mu \mathrm{M}$ NEDD8 variant, $1.6 \mu \mathrm{M}$ full-length Ubc12, $5 \mathrm{mM}$ ATP, 200 ng wild-type NAE, $20 \mathrm{mM}$ Tris pH $7.510 \mathrm{mM} \mathrm{MgCl} 250 \mathrm{mM} \mathrm{NaCl}$. Samples were drawn at indicated time points and reactions were quenched by adding an equal volume of SDS buffer lacking DTT. Samples were directly loaded onto SDS-PAGE gels and products were probed using anti-FLAG monoclonal antibody (Sigma F-3165). Thermal denaturation was monitored at $215 \mathrm{~nm}$ in the presence of $40 \mu \mathrm{M}$ protein, $25 \mathrm{mM}$ Tris $25 \mathrm{mM} \mathrm{NaCl} \mathrm{pH} 7.5$ buffer. Chemical denaturation experiments were performed at $20^{\circ} \mathrm{C}$ in the presence of $50 \mu \mathrm{M}$ protein, $25 \mathrm{mM}$ Tris $25 \mathrm{mM}$ $\mathrm{NaCl}$ pH 7.5 buffer. 
A)

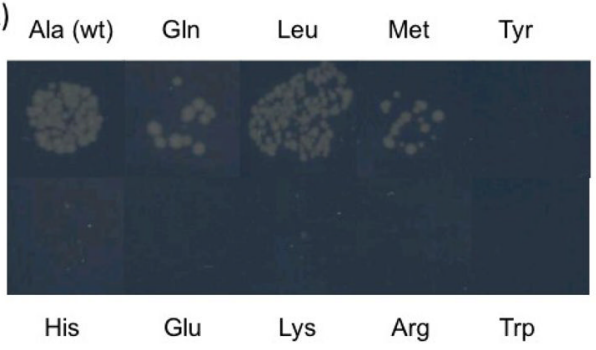

wt Ub $Y$ W $M \quad$ E $\quad H \quad L$
B)

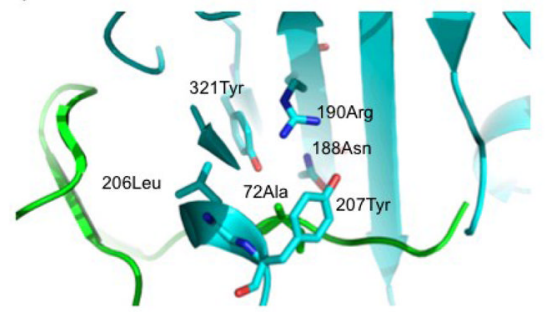

Figure 3.

The screen accurately reflects the effect of mutations at the NEDD8 - NAE interface. A)

Spot test of cells expressing point mutants of NEDD8 at position 72. Western blot of Ubc12 transthiolation (2 minute reaction) with different NEDD8 mutants. Only wt NEDD8 and NEDD8_A72M interacted with wt NAE. Ub: Ubiquitin was tested as negative control. B) Structure of NAE - NEDD8 interface: Five NAE (cyan) residues proximal to Ala72 of NEDD8 (green) were randomized. Sequences of three NAE mutants that interact with NEDD8_A72Y.C) Western blots of Ubc12 transthiolation with w.t or mutant NAE NEDD8 pairs.

To create the site-directed mutagenesis library of NEDD8_Ala72, 4 mutagenic oligonucleotides encoding for 16 amino acid types (proline, glycine, wild-type alanine, and arginine were excluded) were pooled and the NEDD8 gene was PCR amplified and cloned into pNEDD8. Comparison of the sequencing chromatograms for the unselected library plasmid and the plasmid pool after the $2^{\text {nd }}$ round of selection revealed that thymine as the first base of the codon for position 72 was removed from the library. Therefore, we concluded that the selection eliminated plasmids encoding NEDD8_A72(F/Y/W/C/S) mutants. The redesigned NAE library targeted this set of 5 NEDD8 mutants. To create the site-directed mutagenesis library of NAE overlap extension of mutagenic UBA3 fragments were cloned into a library of pNEDD8 plasmids encoding NEDD8_A72(F/ $\mathrm{Y} / \mathrm{W} / \mathrm{C} / \mathrm{S}$ ). Based on the number of colony forming units (CFU) on plates without ampicillin, $\sim 2 \times 10^{6}$ cells (well under the theoretical DNA diversity: $\sim 1.7 \times 10^{8}$ ) were screened on plates supplemented with $32 \mu \mathrm{g} / \mathrm{ml}$ ampicillin. After 2 days of incubation at room temperature, UBA3 gene pool from the survivors were PCR amplified and cloned back into pNEDD8_A72(F/Y/W/C/S). About $10^{6} \mathrm{CFU}$ were screened in the second round of selection under the same conditions as in round 1 . Survivors from round 2 were used to inoculate an overnight culture in the absence of ampicillin before plating a small number of CFU $\left(<10^{4}\right) .4$ clones from the final set of survivors were sequenced. NAE and NAE-2 were purified as described ${ }^{21}$. 